\title{
Thromboembolic events secondary to tirofiban-induced thrombocytopenia being treated with thrombopoietin: A case report
}

\author{
YANHUA LI, QIANG XU and XINHONG GUO \\ Department of Cardiology, Chinese PLA General Hospital, Beijing 100853, P.R. China
}

Received March 12, 2015; Accepted April 7, 2016

DOI: $10.3892 /$ etm.2016.3439

\begin{abstract}
A 68-year-old man presented with acute coronary syndrome (ACS). The glycoprotein/IIb/IIIa receptor antagonist tirofiban was administered to the patient to treat a right coronary artery slow flow detected during percutaneous coronary intervention. The patient developed very severe thrombocytopenia $8 \mathrm{~h}$ after tirofiban infusion with no signs of bleeding. Antiplatelet medication was discontinued immediately, methylprednisolone was administered and platelets were transfused. Thrombopoietin (TPO) was also applied to the patient as recommended by a hematology consultant. As a result, the patient had in-stent thrombosis and cerebral infarction, then another sirolimus-eluting stent was implanted in the proximal right coronary artery and intensive anti-platelet as well as intermittent dehydration to reduce intracranial pressure, and protection of the nervous system function treatment was also provided. The patient was left with a decreased muscle strength in the right limb muscle and fine movement disorder, which may have lead to a poor prognosis. To the best of our knowledge, this is the first case report of tirofiban-induced severe thrombocytopenia with secondary in-stent thrombosis and cerebral infarction from China. The experience of the diagnosis and treatment of this case may shed some light on the clinical use of TPO in tirofiban-induced thrombocytopenia.
\end{abstract}

\section{Introduction}

The platelet membrane glycoprotein IIb/IIIa receptor antagonist (GPRA) tirofiban is widely used for the treatment of acute coronary syndrome (ACS). However, tirofiban may induce thrombocytopenia in certain patients (1). Prior studies have shown that the incidence of GPRA-induced thrombocytopenia (GIT) is $1-5 \%$ (2), and $0.2-0.5 \%$ for severe GIT (3). Tirofiban

Correspondence to: Dr Xinhong Guo, Department of Cardiology, Chinese PLA General Hospital, 28 Fuxing Road, Beijing 100853, P.R. China

E-mail: 13681061699@163.com

Key words: glycoprotein IIb/IIIa receptor antagonist, tirofiban, thrombocytopenia, thrombopoietin is currently the only available GPRA in China, and the incidence of GIT caused by tirofiban in the Chinese population has not been reported, to the best of our knowledge. Due to a lack of associated guidelines or expert consensus, treatment of GIT is inconsistent. Thrombopoietin (TPO) is one of the most effective thrombocytopenic medication (4). While there are some reports on TPO in the treatment of heparin-induced thrombocytopenia (HIT) $(5,6)$, it is not clear whether TPO can be effectively used for the treatment of GIT (4).

In the present study, a case of tirofiban-induced severe thrombocytopenia, or GIT, with secondary in-stent thrombosis and cerebral infarction following TPO treatment is reported. This example of the diagnosis and treatment of a case of ACS highlights the need for caution and careful monitoring of the clinical use of tirofiban and TPO in patients with ACS and recognition of the risk of tirofiban-induced thrombocytopenia.

\section{Case report}

A 68-year-old man presented in the Department of Cardiology on July 4th, 2012, Chinese PLA General Hospital (Beijing, China), with paroxysmal chest depression of 1-month duration. The patient had a past medical history of hypertension, and was not allergic to any medication. After admission, the patient began to receive dual antiplatelet therapy with aspirin $(100 \mathrm{mg}$, Bayer AG, Leverkusen, Germany) and clopidogrel (75 mg, Sanofi Pharmaceutical Co., Ltd., Hangzhou, China) without heparin (SPH No .1 Biochemical \& Pharmaceutical Co., Ltd., Shanghai). Patient informed consent was obtained for the present study.

Five days after admission, coronary angiography (GE Medical Systems Ltd., Chalfont St Giles, UK) detected a $50 \%$ stenosis at the proximal left anterior descending artery, 30\% stenosis at the proximal circumflex branch, and $90 \%$ stenosis at the mid-portion of the right coronary artery. A sirolimus-eluting stent $\left(3.5 \times 21 \mathrm{~mm}\right.$; Cypher ${ }^{\mathrm{TM}}$; Cordis Corporation, Bridgewater Township, NJ, USA) was implanted at the right coronary artery and the patient received a total of 10,500 units of heparin (SPH No. 1 Biochemical \& Pharmaceutical Co., Ltd.,). During the surgical procedure, the patient displayed coronary slow flow, and tirofiban $(8 \mathrm{ml} / \mathrm{h}$, total $0.4 \mathrm{mg}$; Great Medical China Co., Ltd., Wuhan, Hubei, China) was administered intravenously. At $8 \mathrm{~h}$ after the 


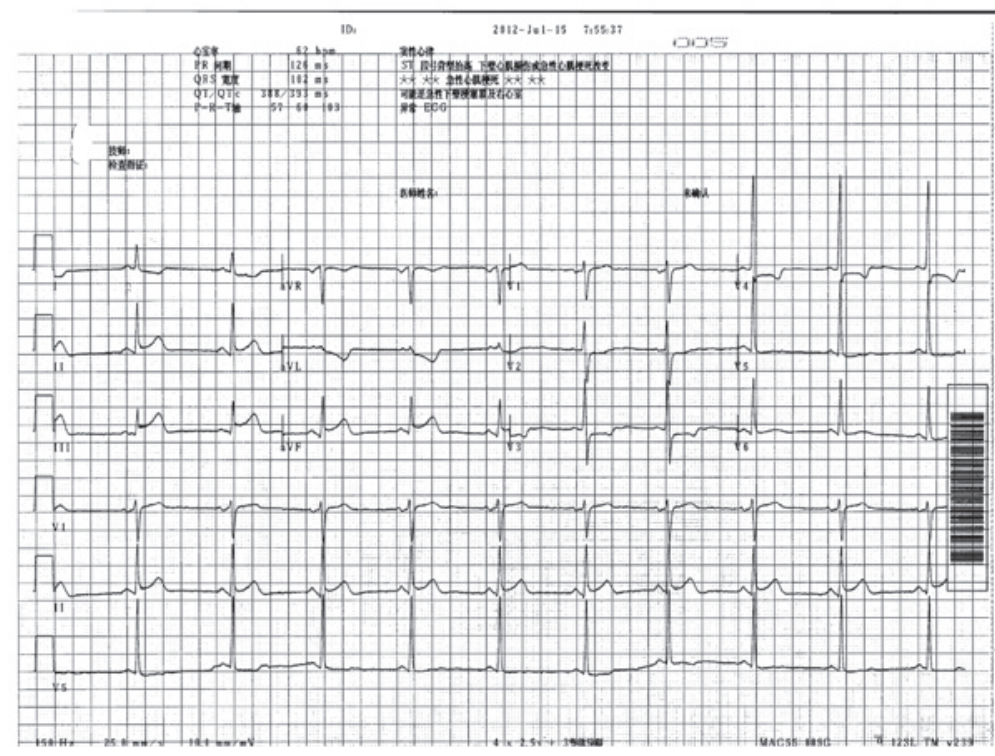

Figure 1. Electrocardiogram of a 68-year-old man showing acute inferior wall myocardial infarction after 7 days of glycoprotein IIb/IIIa receptor antagonist-induced thrombocytopenia and 3 days after the discontinuation of thrombopoietin.
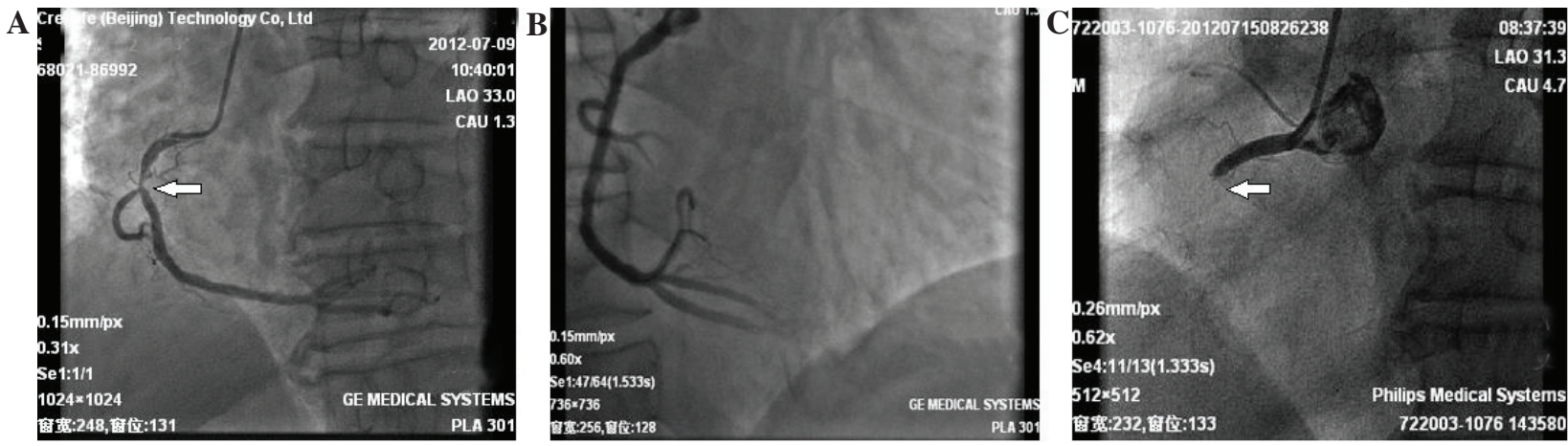

Figure 2. Coronary angiography. (A) Approximately 90\% stenosis of the right coronary artery (arrow) was observed. (B) A drug-eluting stent was successfully implanted. (C) Right coronary acute stent thrombosis and total occlusion of the right coronary (arrow).

procedure, the platelet count of the patient decreased from $234 \times 10^{9} / 1$ to $10 \times 10^{9} / 1$, while hemoglobin levels, clotting function, and hepatic and renal functions were normal. When the platelet count was repeated, manually, a further reduction to $3 \times 10^{9} / 1$ was noted. All antiplatelet and antithrombotic agents were discontinued immediately, and following consultation with a hematologist, $40 \mathrm{mg}$ methylprednisolone (Pfizer Inc., New York, NY, USA) and 1 unit platelets were administered intravenously, and 15,000 units TPO (3S Bio Inc., Hong Kong, China) were injected subcutaneously once daily. The platelet count rose to $34 \times 10^{9} / 1$ after $48 \mathrm{~h}$ and to $71 \times 10^{9} / 1$ after 4 days of treatment. TPO was discontinued (total of 60,000 IU), and aspirin, clopidogrel and a low-molecular-weight heparin (60 mg, twice a day, Sanofi Pharmaceutical Co., Ltd.) therapy were administered.

On the seventh morning of GIT and 3 days after the discontinuation of TPO, the patient complained of acute chest pain. Electrocardiography (MAC 5000 ECG Analysis System, GE Medical Systems Ltd.) suggested acute inferior wall myocardial infarction (Fig. 1). The platelet count was 179x10\%1 (normal range: $100-300 \times 10^{9} / 1$ ), and the platelet aggregation test showed a value of $62 \%$ (normal range: $20-60 \%$, calculated

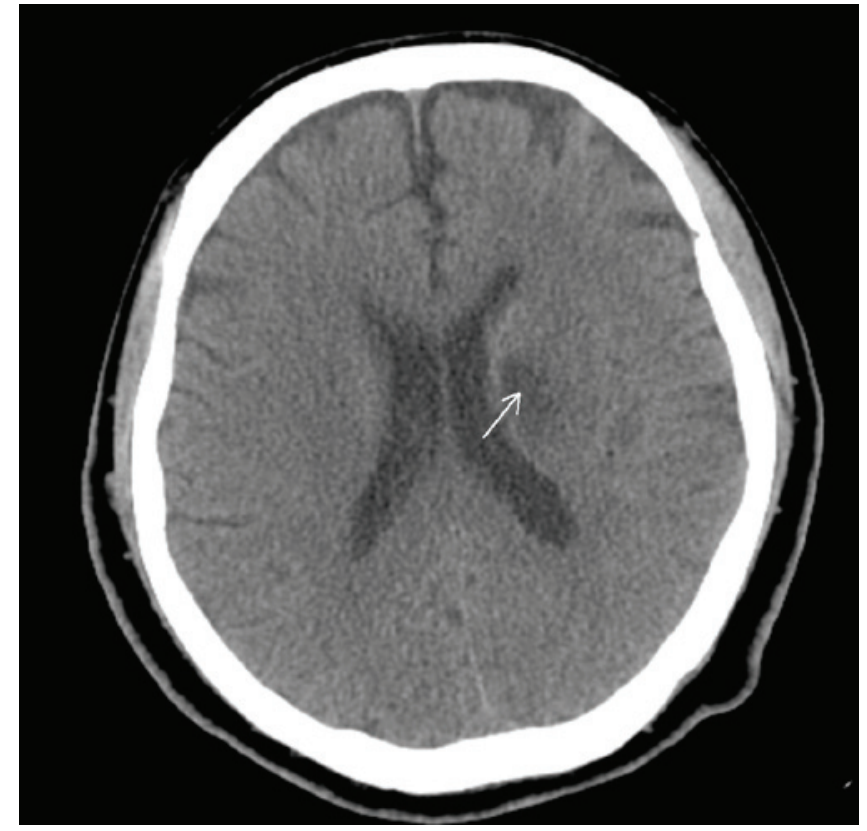

Figure 3. Computed tomography scan showing cerebral capsule infarction of the left part of the brain (arrow). 


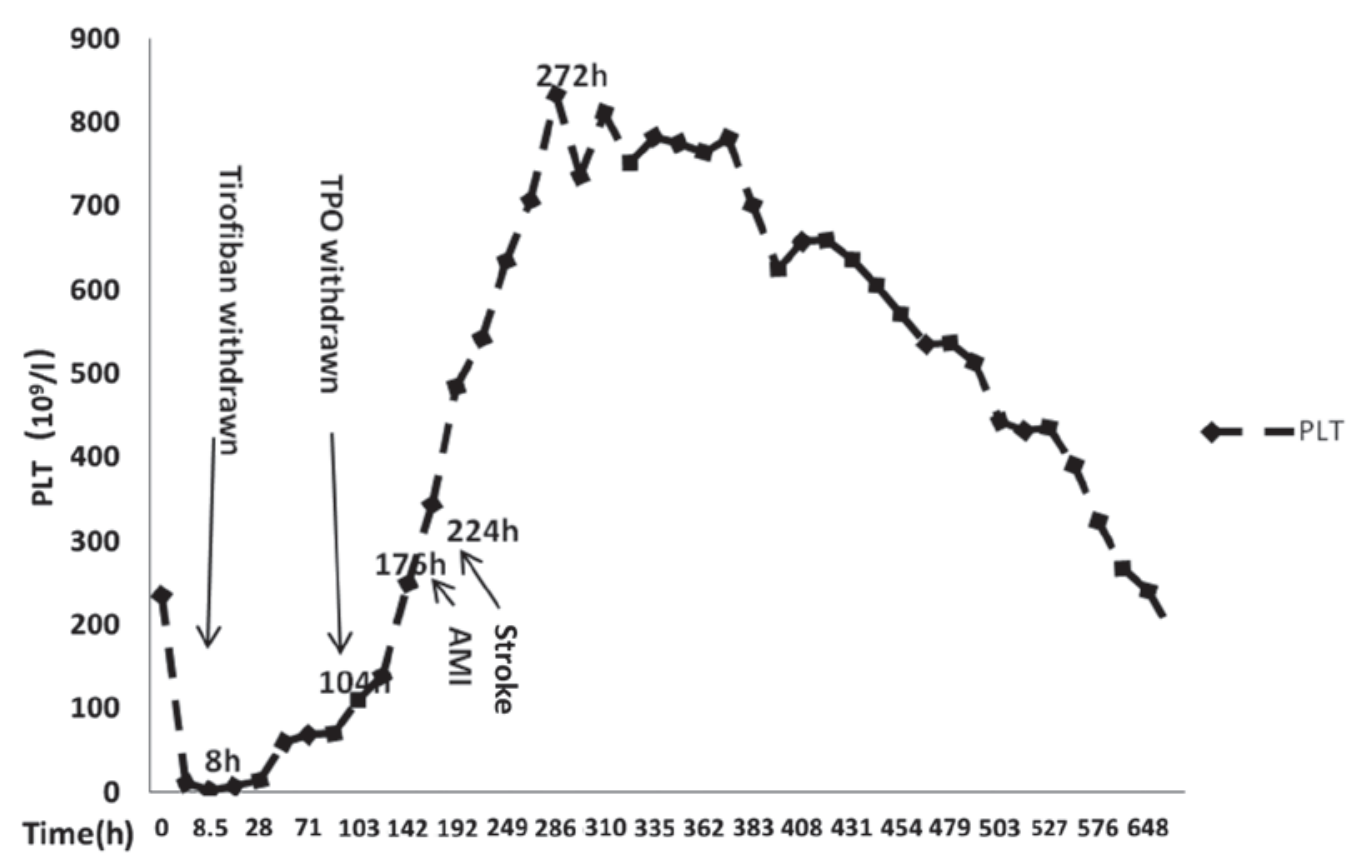

Figure 4. Change in platelet (PLT) count over time. A 68-year-old man presented with acute coronary syndrome and tirofiban was administered (0 h). Glycoprotein IIb/IIIa receptor antagonist-induced thrombocytopenia (GIT) occurred after $8 \mathrm{~h}$ of tirofiban use ( $8 \mathrm{~h}$ ), then thrombopoietin (TPO) was administered and tirofiban was withdrawn. TPO was withdrawn at $104 \mathrm{~h}$, and at $176 \mathrm{~h}$ inferior wall myocardial infarction (AMI) occurred and cerebral infarction (stroke) occurred at $224 \mathrm{~h}$ of GIT. The PLT count peaked at $272 \mathrm{~h}$ and declined until measurement was discontinued at $696 \mathrm{~h}$.

using Turbidimetric platelet aggregometry, aggregation remote analyzer module, Helena Laboratories, Beaumont, TX, USA). In brief, the procedure is as follows: Blood specimen is collected and handled. The aggregation reagents are reconstituted according to the directions of the manual. ADP, epinephrine and collagen reagents should be used undiluted and will have the final concentrations given below when mixed with the PRP during testing. The aggregometer was prepared for use and a $450 \mu \mathrm{l}$ pipette of platelet poor plasma (PPP) was added to a cuvette. This blank was used to set the $100 \%$ aggregation value. The cuvette was incubated at $37^{\circ} \mathrm{C}$ between one and three minutes. Next, the PPP cuvette was inserted into the appropriate channel and the instrument was set to $100 \%$ aggregation, and the cuvette set into the appropriate channel. Next, $50 \mu \mathrm{l}$ of the aggregating reagent dilutions were added to the PRP cuvette and the percentage of aggregation was recorded (instrument sets $0 \%$ when the aggregation agent is added and the channel activated).

Following intensified antiplatelet treatment with $300 \mathrm{mg}$ clopidogrel and $100 \mathrm{mg}$ aspirin, a repeat coronary angiography (Philips Medical Systems, Eindhoven, Holland) was performed, which showed in-stent thrombosis and total occlusion of the right coronary artery (Fig. 2). After thrombus aspiration, another sirolimus-eluting stent $(3.5 \times 33 \mathrm{~mm}$; Firebird; MicroPort Medical Group, Co., Ltd., Shanghai, China) was implanted, and a Thrombolysis In Myocardial Infarction blood flow at level 3 was restored. Dual anti-platelet therapy with $100 \mathrm{mg}$ of aspirin and $150 \mathrm{mg}$ of clopidogrel was applied. Heparin was injected intravenously for anticoagulation.

On day 9 after GIT and 5 days after the discontinuation of TPO, the platelet count rose to $314 \times 10^{9} / 1$, and the platelet aggregation rate was $54 \%$. The patient had dysarthrosis, flatness of the right nasolabial groove, and a deteriorated pain sensation; the muscle force of his right-sided extremities became $<5^{\circ}$. An emergency cranial computed tomography (CT; Siemens dual-source computer tomography, Munich, Germany) scan ruled out cerebral hemorrhage, and no definite ischemic lesion was found. As these symptoms appeared on the third day after coronary intervention, cerebral embolism caused by plaque shedding during the procedure could be ruled out. Acute cerebral infarction was confirmed by cranial CT 1 week later (Fig. 3). The platelet count increased progressively, and 11 days after the initiation of GIT treatment (7 days after discontinuing TPO), it leveled out at $834 \times 10^{9} / 1$, while the platelet aggregation rate was 24\%. Hydroxycarbamide (1 g, twice daily; (Shandong Qilu, Xinghua, China) was applied to reduce the platelet count. After administering hydroxycarbamide for 7 days, the platelet count was gradually reduced to $636 \times 10^{9} / 1$, and hydroxycarbamide was discontinued. Antiplatelet treatment with aspirin $(100 \mathrm{mg})$ and clopidogrel $(150 \mathrm{mg})$ daily and anticoagulant treatment was continued with fondaparinux sodium (2.5 mg; GlaxoSmithKline, Cedex, France).

At 10 days after the withdrawal of hydroxycarbamide, when the platelet count was reduced to $186 \times 10^{9} / 1$ (Fig. 4), the patient was discharged. At the 28-month follow-up examination, the patient's medical condition was stable.

\section{Discussion}

The mechanism of GIT remains unclear, but may be associated with an autoimmune reaction (5). According to this theory, a GRPA can alter the configuration of the platelet glycoprotein receptor, thereby creating a new antigenic determinant. This antigen is recognized by serum antibodies, leading to the removal of thrombocytes from the blood circulation. The 
prognosis for patients with GIT is poor (7). Evaluation of abciximab (c7E3 Fab), a monoclonal antibody that binds to the platelet glycoprotein IIb/IIIa receptor and inhibits platelet aggregation, in the Prevention of Ischemic Complications (EPIC) study (8) showed that patients with GIT have a high risk of mortality, myocardial infarction and revascularization. In the present case, the patient received multiple antithrombotic agents, such as aspirin, clopidogrel, unfractionated heparin and tirofiban prior to thrombocytopenia. After analyzing the case, and considering that the clinical diagnostic criteria of GIT are primarily based on the relationship between the timing of medical administration and thrombocytopenia, a clear conclusion could be made that the patient's thrombocytopenia was induced by tirofiban. Since heparin was administered during the stent implantation procedure, heparin-induced thrombocytopenia could be ruled out. The patient had no past history of heparin contact, and thrombocytopenia developed fairly early and severely, which was not compliant with the typical characteristics of type I or type II HIT (9). In addition, following the discontinuation of tirofiban, the platelet count reverted to the normal range. During the procedure of revascularization of the right coronary artery, the large dosage of heparin did not trigger thrombocytopenia. Therefore, it is likely that in the present case thrombocytopenia was induced by tirofiban.

TPO is an effective thrombocytopenic medication that may act directly on bone marrow hematopoietic stem cells to specifically raise platelet counts (10). However, the effect of TPO starts slowly; the platelet count starts to rise at 4-9 days, and reaches a peak at 12-16 days after subcutaneous injection (8). Since the patient with GIT in the present study had normal bone marrow function, when the effect of TPO started, the patient theoretically might have already recovered from GIT, and therefore TPO could have promoted a further rise in platelet numbers, thus increasing the possibility of thrombosis. However, when the thrombotic events occurred, the platelet count was relatively low within the normal range. It is difficult to determine whether the thrombotic events were drug-related, but the use of TPO may have been an important contributing factor.
In summary, from this case, the conclusion can be drawn that administration of tirofiban should be conducted under careful clinical monitoring in order to detect GIT as early as possible. GIT usually develops within $24 \mathrm{~h}$ after tirofiban administration, and the platelet count resumes to a normal range 1-6 days after discontinuation of tirofiban (11). In the present case, it was critically important to discontinue tirofiban once GIT was detected. Moreover, in view of the possible thrombotic risk of TPO application, it should be cautiously used in the treatment of GIT.

\section{References}

1. Dasgupta H, Blankenship JC, Wood GC, Frey CM, Demko SL and Menapace FJ: Thrombocytopenia complicating treatment with intravenous glycoprotein IIb/IIIa receptor inhibitors: A pooled analysis. Am Heart J 140: 206-211, 2000.

2. Yeung J and Holinstat M: Newer agents in antiplatelet therapy: A review. J Blood Med 3: 33-42, 2012.

3. Labinaz M, Ho C, Banerjee S, Martin J, Chen S and Mensinkai S: Meta-analysis of clinical efficacy and bleeding risk with intravenous glycoprotein IIb/IIIa antagonists for percutaneous coronary intervention. Can J Cardiol 23: 963-970, 2007.

4. Li B, Ji Y, Shao Q, Zhu Z, Ji D, Li F and Chen G. Comparative efficacy and cost effectiveness of splenectomy and thrombopoietin prior to peginterferon and ribavirin therapy with compensatory cirrhosis associated with hepatitis $\mathrm{C}$ and thrombocytopenia. Exp Ther Med 10: 2180-2186, 2015.

5. Gudbrandsdottir S, Frederiksen H, Birgens HS, Nielsen $\mathrm{CH}$, Nielsen OJ, Stentoft J and Hasselbalch HC: New treatment options for primary immune thrombocytopenia. Ugeskr Laeger 173: 271-274, 2011 (In Danish).

6. Yuan SM: Report: Heparin-induced thrombocytopenia associated with cardiopulmonary bypass: Preliminary attempt with recombinant human thrombopoietin therapy. Pak J Pharm Sci 28: 1793-1796, 2015.

7. Tuhta AG, Yeşildağ $\mathrm{O}$ and Köprülü D: Tirofiban-associated acute thrombocytopenia. Acta Cardiol 61: 577-579, 2006.

8. Kuter DJ: The biology of thrombopoietin and thrombopoietin receptor agonists. Int J Hematol 98: 10-23, 2013.

9. Huxtable LM, Tafreshi MJ and Rakkar AN. Frequency and management of thrombocytopenia with the glycoprotein IIb/IIIa receptor antagonists. Am J Cardiol 97: 426-429, 2006.

10. Kuter DJ: Thrombopoietin: Biology and clinical applications Oncologist 1: 98-106, 1996

11. Aster RH: Immune thrombocytopenia caused by glycoprotein IIb/IIIa inhibitors. Chest 127 (2 Suppl): 53S-59S, 2005. 